\title{
Towards a resolved Kennicutt-Schmidt law at high redshift
}

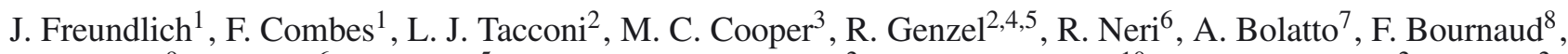 \\ A. Burkert ${ }^{9, \star}$, P. Cox $^{6}$, M. Davis ${ }^{5}$, N. M. Förster Schreiber ${ }^{2}$, S. Garcia-Burillo ${ }^{10}$, J. Gracia-Carpio ${ }^{2}$, D. Lutz ${ }^{2}$, \\ T. Naab ${ }^{11}$, S. Newman ${ }^{5}$, A. Sternberg ${ }^{12}$, and B. Weiner ${ }^{13}$ \\ ${ }^{1}$ LERMA, Observatoire de Paris, CNRS, 61 Av. de l'Observatoire, 75014 Paris, France \\ e-mail: jonathan. freundlich@obspm.fr \\ 2 Max-Planck-Institute für extraterrestrische Physik (MPE), Giessenbachstrasse 1, 85748 Garching, Germany \\ 3 Dept. of Physics \& Astronomy, Frederick Reines Hall, University of California, Irvine, CA 92697, USA \\ ${ }^{4}$ Dept. of Physics, Le Conte Hall, University of California, CA 94720 Berkeley, USA \\ 5 Dept. of Astronomy, Campbell Hall, University of California, CA 94720 Berkeley, USA \\ 6 IRAM, 300 rue de la Piscine, 38406 St. Martin d'Hères, Grenoble, France \\ 7 Dept. of Astronomy, University of Maryland, College Park, MD 20742-2421, USA \\ 8 CEA, IRFU, SAp, 91191 Gif-sur-Yvette, France \\ 9 Universitätsternwarte der Ludwig-Maximiliansuniversität, Scheinerstrasse 1, 81679 München, Germany \\ 10 Observatorio Astronómico Nacional - OAN, Apartado 1143, 28800 Alcalá de Henares, Madrid, Spain \\ 11 Max Planck Institut für Astrophysik, Karl Schwarzshildstrasse 1, 85748 Garching, Germany \\ 12 School of Physics and Astronomy, Tel Aviv University, 69978 Tel Aviv, Israel \\ 13 Steward Observatory, 933 N. Cherry Ave., University of Arizona, Tucson, AZ 85721-0065, USA
}

Received 21 December 2012 / Accepted 24 March 2013

\begin{abstract}
Massive galaxies in the distant Universe form stars at much higher rates than today. Although direct resolution of the star forming regions of these galaxies is still a challenge, recent molecular gas observations at the IRAM Plateau de Bure interferometer enable us to study the star formation efficiency on subgalactic scales around redshift $z=1$. We present a method for obtaining the gas and star formation rate (SFR) surface densities of ensembles of clumps composing galaxies at this redshift, even though the corresponding scales are not resolved. This method is based on identifying these structures in position-velocity diagrams corresponding to slices within the galaxies. We use unique IRAM observations of the $\mathrm{CO}(3-2)$ rotational line and DEEP2 spectra of four massive star forming distant galaxies - EGS13003805, EGS13004291, EGS12007881, and EGS13019128 in the AEGIS terminology - to determine the gas and SFR surface densities of the identifiable ensembles of clumps that constitute them. The integrated CO line luminosity is assumed to be directly proportional to the total gas mass, and the SFR is deduced from the [OII] line. We identify the ensembles of clumps with the angular resolution available in both $\mathrm{CO}$ and [OII] spectroscopy; i.e., 1-1.5". SFR and gas surface densities are averaged in areas of this size, which is also the thickness of the DEEP2 slits and of the extracted IRAM slices, and we derive a spatially resolved Kennicutt-Schmidt (KS) relation on a scale of $\sim 8 \mathrm{kpc}$. The data generally indicates an average depletion time of 1.9 Gyr, but with significant variations from point to point within the galaxies.
\end{abstract}

Key words. galaxies: evolution - galaxies: high-redshift - galaxies: structure - stars: formation - galaxies: ISM - galaxies: starburst

\section{Introduction}

Ten billion years ago, between redshifts 1 and 3, observed galaxies formed their stars at rates as high as ten times that of the Milky Way today (Noeske et al. 2007; Daddi et al. 2007). This implies a more abundant gas supply, either fueled by major mergers or by a semi-continuous gas accretion (Dekel et al. 2009a,b). Recent observations of normal, massive, star-forming distant galaxies tend to show that most of these galaxies $(\geqslant 80 \%)$ are not experiencing major mergers or interactions (Tacconi et al. 2013) and that their light profiles are similar to those of their low redshift counterparts (Wuyts et al. 2011b). Also, the KennicuttSchmidt (KS) relation between total molecular gas and star formation rate (SFR) surface densities is nearly linear at all redshifts (Kennicutt 1998a; Wyder et al. 2009; Genzel et al. 2010; Tacconi et al. 2010), implying that the star formation processes

\footnotetext{
* MPG-Fellow at MPE.
}

seem to be largely independent of the cosmic epoch. The average fraction of molecular gas relative to the total baryonic mass must therefore have been up to ten times higher at high redshift than in today's nearby galaxies, which is indeed corroborated by direct observations (Tacconi et al. 2013).

Numerical simulations suggest that typical massive star forming galaxies at high redshift continually accrete gas from the intergalactic medium along cold and clumpy streams stemming from the cosmic web, while the disk fragments into a few clumps (Kereš et al. 2005; Bournaud \& Elmegreen 2009; Dekel et al. 2009a; Van de Voort et al. 2011). Galactic disks of distant galaxies are indeed found to be fragmented in a number of giant molecular clouds, or clumps, which differs from local galaxies, in which the molecular gas is scattered in numerous lower mass giant molecular clouds. The high-redshift star-forming complexes are found to have typical scales of $\sim 1 \mathrm{kpc}$ and masses up to $10^{9} M_{\odot}$, and they contribute $10-25 \%$ of the galaxy luminosity (Förster Schreiber et al. 2011). Most 

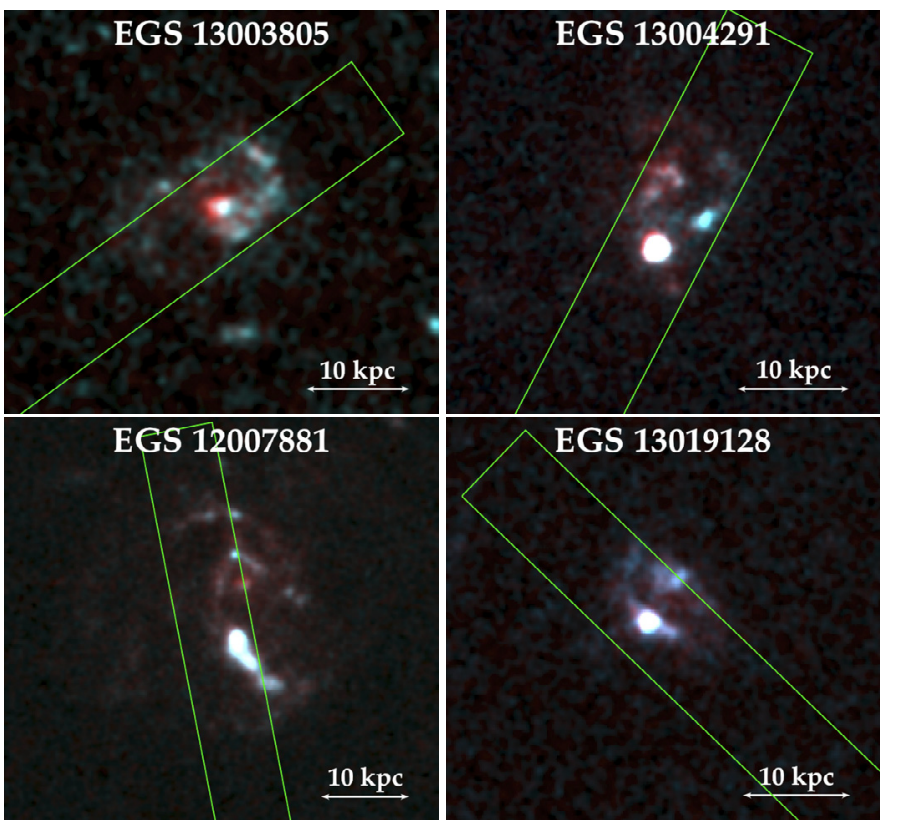

Fig. 1. Composite HST images of the four galaxies studied here, combining ACS $I$ and $V$ bands. The green box shows the DEEP2 slit used for each galaxy, whose size is indicated in Table 1. We assumed that the DEEP2 survey measured the position of the center of the galaxy more accurately than the HST, and shifted the HST image accordingly. North is up, east is left.

studies only consider averaged quantities for distant galaxies, as direct observations of the star forming regions of high redshift galaxies are still challenging. KS relations have been derived on subgalactic scales for nearby galaxies (Bigiel et al. 2008, 2011; Leroy et al. 2013) but not yet extensively at high redshift.

We use IRAM Plateau de Bure CO observations from the PHIBSS survey (Tacconi et al. 2013) and Keck DEEP2 spectra (Newman et al. 2012) of four massive galaxies at redshift $z \sim 1.2$ in order to investigate the star formation efficiency within their clumps, or groups of clumps. More precisely, our goal is to determine the SFR and the gas mass surface densities on subgalactic scales and examine their correlation.

The four galaxies were drawn from the All-wavelength Extended Groth strip International Survey (AEGIS), which provides deep imaging in all major wave bands from X-rays to radio, including Hubble Space Telescope (HST) images, and optical spectroscopy (DEEP2/Keck) over a large area of sky (Noeske et al. 2007; Davis et al. 2007; Cooper et al. 2011, 2012; Newman et al. 2012), thus providing a complete set of galaxies between $0.2 \leqslant z \leqslant 1.2$. The four galaxies studied here were selected from the sample analyzed by Tacconi et al. (2010, 2013), which gathered non-major-merger, luminous, star forming galaxies at $z \sim 1.2$, with stellar masses above $3 \times 10^{10} M_{\odot}$ and SFR above $40 M_{\odot} \mathrm{yr}^{-1}$. These four galaxies - named EGS13003805, EGS13004291, EGS12007881, and EGS13019128 in the AEGIS terminology - correspond to the massive end of the "normal" star forming galaxies at $z \sim 1.2$ (Tacconi et al. 2010). Figure 1 shows them in the $I$ and $V$ bands of the HST Advanced Camera for Surveys (ACS), as well as the DEEP2 slits we used. To compute the physical distances, we adopt a cosmology with $\Omega_{\Lambda}=0.73, \Omega_{\mathrm{m}}=0.27$, and $H_{0}=70 \mathrm{~km} \mathrm{~s}^{-1} \mathrm{Mpc}^{-1}$.

\section{Determination of the total gas mass and the SFR}

\subsection{The gas mass}

We use high resolution observations of the $\mathrm{CO}(3-2)$ transition shifted into the $2 \mathrm{~mm}$ band for $z \sim 1.2$ sources carried at the IRAM Plateau de Bure, including the most extended "A" configuration. The angular resolution obtained is between 0.5 " and $1.5^{\prime \prime}$ for all galaxies (Table 1), corresponding to physical scales between 4 and $13 \mathrm{kpc}$. The CO luminosity associated to any region of flux can be expressed as

$$
\left(\frac{L_{\mathrm{CO}}^{\prime}}{\mathrm{K} \mathrm{km} \mathrm{s}^{-1} \mathrm{pc}^{2}}\right)=3.25 \times 10^{7}(1+z)^{-3}\left(\frac{S_{\mathrm{CO}} \Delta V}{\mathrm{Jy} \mathrm{km} \mathrm{s}^{-1}}\right)\left(\frac{v_{\mathrm{obs}}}{\mathrm{GHz}}\right)^{-2}\left(\frac{D_{L}}{\mathrm{Mpc}}\right)^{2}
$$

where $S_{\mathrm{CO}} \Delta V$ is the velocity integrated flux, $v_{\mathrm{obs}}$ the observed frequency, and $D_{L}$ the luminosity distance (Solomon et al. 1997). To derive $\mathrm{H}_{2}$ masses, we follow the approach used by Tacconi et al. (2010). This assumes a Milky-Way-like conversion factor of $\alpha=3.2 M_{\odot}\left(\mathrm{K} \mathrm{km} \mathrm{s}^{-1} \mathrm{pc}^{2}\right)^{-1}$ between the $\mathrm{CO}(1-0)$ luminosity and the $\mathrm{H}_{2}$ mass, a factor 1.36 to account for interstellar helium, and a further correction by a factor 2 for the $\mathrm{CO}(3-2) / \mathrm{CO}(1-0)$ luminosity ratio. The total gas masses of the four studied galaxies are indicated in Table 1.

\subsection{The star formation rate}

The $\mathrm{H} \alpha$ recombination line is the most direct and reliable tracer of young massive stars, and thus of the SFR (Kennicutt 1998b). Since this line lies in the middle of a low atmospheric transmission band for the four galaxies considered here, groundbased $\mathrm{H} \alpha$ spectroscopy is impossible, and we estimate the SFR from the collisionally excited [OII] line (rest frame wavelength $3727 \AA$, shifted around $8200 \AA$ for $z \sim 1.2$ sources).

Though the [OII] forbidden line luminosity is not directly coupled to the ionizing luminosity, it is possible to establish it empirically as a quantitative SFR tracer. The Kennicutt (1998b) [OII] SFR calibration has been broadly used, but does not take reddening and metallicity into account. Here we use the calibration proposed by Kewley et al. (2004), which includes these two effects. The four galaxies studied here are particularly massive and should thus belong to the observed metallicity plateau at $12+\log (\mathrm{O} / \mathrm{H})=9.07$ (Mannucci et al. 2010). Assuming this metallicity for all galaxies, the Kewley et al. (2004) [OII] SFR calibration gives

$$
\left(\frac{\mathrm{SFR}}{M_{\odot} \mathrm{yr}^{-1}}\right) \simeq 3.5 \times 10^{-8}\left(\frac{L_{[\mathrm{OII}]}}{L_{\odot}}\right)
$$

where $L_{[\mathrm{OII}]}$ is the intrinsic [OII] line luminosity. This calibration is expected to follow the reliable standard Kennicutt (1998b) $\mathrm{H} \alpha$ SFR calibration with a scatter of 0.03-0.05 dex (Kewley et al. 2004).

To determine $L_{[\mathrm{OII}]}$, we use spectra provided by the Keck DEEP2 survey, obtained with the high spectral resolution DEIMOS spectrograph (Faber et al. 2002) and taken along slits with a typical size of $5^{\prime \prime} \times 1^{\prime \prime}$. The data was reduced using a dedicated pipeline (Newman et al. 2012; Cooper et al. 2012), and a velocity resolution of about $50 \mathrm{~km} \mathrm{~s}^{-1}(R=\lambda / \Delta \lambda \approx 5000)$ enables resolving the $[\mathrm{OII}]$ line (Davis et al. 2003). DEEP2 spectra are not flux-calibrated, and we use CFHT I band magnitudes (CFH12K camera) to calibrate the galaxy-integrated "1D" spectrum (Coil et al. 2004).

The value of $L_{[\mathrm{OII}]}$ has to be corrected for dust extinction. The Galactic extinction is taken into account in the CFHT 
Table 1. Some global properties and observational details for the four galaxies studied here.

\begin{tabular}{lcccc}
\hline \hline Properties & EGS 13003805 & EGS 13004291 & EGS 12007881 & EGS 13019128 \\
\hline$z^{1}$ & 1.2318 & 1.19705 & 1.16105 & 1.3494 \\
$D_{A}[\mathrm{Mpc}]^{2}$ & 1753.0 & 1745.3 & 1736.4 & 1772.8 \\
$D_{L}[\mathrm{Mpc}]^{2}$ & 8731.5 & 8424.7 & 8108.8 & 9785.1 \\
Scale $\left.[\mathrm{kpc})^{\prime \prime}\right]$ & 8.50 & 8.46 & 8.42 & 8.60 \\
\hline DEEP2 slit size & $6.863^{\prime \prime} \times 1^{\prime \prime}$ & $5.149^{\prime \prime} \times 1^{\prime \prime}$ & $5.762^{\prime \prime} \times 1^{\prime \prime}$ & $10.343^{\prime \prime} \times 1^{\prime \prime}$ \\
DEEP2 slit orientation $(\mathrm{PA})$ & $-53.858^{\circ}$ & $-27.649^{\circ}$ & $11.142^{\circ}$ & $46.142^{\circ}$ \\
$\mathrm{IRAM} \mathrm{CO}(3-2) \mathrm{beam}^{\prime \prime}$ & $0.8^{\prime \prime} \times 0.6^{\prime \prime}$ & $0.6^{\prime \prime} \times 0.5^{\prime \prime}$ & $1.1^{\prime \prime} \times 0.9^{\prime \prime}$ & $1.6^{\prime \prime} \times 1.4^{\prime \prime}$ \\
\hline $\mathrm{SFR}_{[\mathrm{OII}]}\left[M_{\odot} \mathrm{yr}^{-1}\right]^{3}$ & 98 & 182 & 119 & 202 \\
$\mathrm{SFR}_{\mathrm{UV}+24 \mu \mathrm{m}}\left[M_{\odot} \mathrm{yr}^{-1}\right]^{4}$ & 200 & 630 & 94 & 87 \\
$\mathrm{SFR}_{[\mathrm{OIII}, \mathrm{B}}\left[M_{\odot} \mathrm{yr}^{-1}\right]^{5}$ & 63 & 231 & 265 & 213 \\
\hline$M_{\text {gas }}\left[10^{11} M_{\odot}\right]^{6}$ & 2.2 & 2.8 & 1.3 & 1.2 \\
$M_{\text {star }}\left[10^{11} M_{\odot}\right]^{7}$ & 3.4 & 5.0 & 5.0 & 3.8 \\
$\mathrm{f}_{\mathrm{gas}}{ }^{3}$ & 0.39 & 0.36 & 0.21 & 0.24 \\
\hline$R_{1 / 2}[\mathrm{kpc}]^{9}$ & 5.7 & 3.1 & 5.7 & 5.2 \\
$\log _{10}\left(\Sigma_{\mathrm{gas}} /\left[M_{\odot} \mathrm{pc}^{-2}\right]\right)^{10}$ & 3.03 & 3.67 & 2.80 & 2.86 \\
$\log _{10}\left(\Sigma_{\mathrm{SFR}} /\left[M_{\odot} \mathrm{yr}^{-1} \mathrm{kpc}^{-2}\right]\right)^{10}$ & -0.193 & 0.604 & -0.095 & 0.204 \\
$\log _{10}\left(\Sigma_{\mathrm{star}} /\left[M_{\odot} \mathrm{pc}^{-2}\right]\right)^{10}$ & 3.22 & 3.92 & 3.39 & 3.36 \\
\hline$A_{\mathrm{H} \alpha}{ }^{11}$ & 1.25 & 1.28 & 1.06 & 1.21 \\
$A_{V}{ }^{11}$ & 1.56 & 1.60 & 1.30 & 1.50 \\
$A_{[\mathrm{OIII}]}{ }^{11}$ & 2.28 & 2.26 & 2.04 & 2.19 \\
$A_{[\mathrm{OII}]} / A_{\mathrm{H} \alpha}{ }^{12}$ & 1.82 & 1.77 & 1.92 & 1.81 \\
\hline
\end{tabular}

Notes. ${ }^{(1)}$ The redshift, as determined in the AEGIS catalogues from the [OII] line using DEEP2 spectra; ${ }^{(2)}$ the angular distance $D_{A}$ and the luminosity distance $D_{L} ;{ }^{(3)}$ the SFR from the [OII] line luminosity in the galaxy-integrated "1D" spectrum, obtained according to Kewley et al. (2004) calibration; ${ }^{(4)}$ the extinction-corrected SFR obtained from $24 \mu \mathrm{m}$ and UV continuum by Tacconi et al. (2013); ${ }^{(5)}$ the SFR extrapolated from the empirical calibration of Moustakas et al. (2006); ${ }^{(6)}$ the gas mass derived from the IRAM Plateau de Bure observations; ${ }^{(7)}$ the total star mass obtained by SED fittings from CFHT $B, R$, and $I$ bands photometric data using kcorrect (Blanton \& Roweis 2007), corrected for a Universe with $H_{0}=70 \mathrm{~km} \mathrm{~s}^{-1} \mathrm{Mpc}^{-1}$; ${ }^{(8)}$ the gas fraction $f_{\mathrm{gas}}=M_{\mathrm{gas}} /\left(M_{\mathrm{gas}}+M_{\mathrm{star}}\right)$; ${ }^{(9)}$ the optical half light radius $R_{1 / 2}$ as indicated in Tacconi et al. (2013); ${ }^{(10)}$ the surface densities associated to the gas mass, the star formation rate, and the star mass, calculated within $R_{1 / 2}$, for example as $\Sigma_{\text {gas }}=0.5 M_{\text {gas }} / \pi R_{1 / 2}^{2},{ }^{(11)} \mathrm{H} \alpha$, visible, and [OII] extinctions as derived from the kcorrect SED reconstruction; and ${ }^{(12)}$ the extinction ratio $A_{\mathrm{H} \alpha} / A_{[\mathrm{OII}]} . A_{V}, A_{[\mathrm{OII}]}, A_{\mathrm{H} \alpha} / A_{[\mathrm{OII}]}, \mathrm{SFR}_{24 \mu \mathrm{m}+\mathrm{UV}}$ and $\mathrm{SFR}_{[\mathrm{OII}], \mathrm{B}}$ are given for information only. O'Donnell (1994) predicts that $A_{\mathrm{H} \alpha} / A_{[\mathrm{OII}]}$ is around 1.86 for a diffuse interstellar medium, and the variations here observed from one galaxy to the other are representative of the quality of the kcorrect fit. The comparison between $\mathrm{SFR}_{[\mathrm{OII}]}, \mathrm{SFR}_{24 \mu \mathrm{m}+\mathrm{UV}}$, and $\mathrm{SFR}_{[\mathrm{OII}], \mathrm{B}}$ gives an idea of the uncertainties in the SFR.

magnitudes, since they already include the dust corrections of Schlegel et al. (1998), but the dust present in the galaxies themselves is an even greater cause of extinction. The dust distribution was derived from the SED of the galaxies, obtained from the $B, R$, and $I$ band CFHT magnitudes with the kcorrect software developed by Blanton \& Roweis (2007) (version v4_2). This software finds the best non-negative linear combination of a number of template star formation histories fitting the CFHT magnitudes. The templates are based on stellar population synthesis models, which enables the software to provide approximate physical quantities related to the star formation history. It notably provides SED curves with and without extinction, from which we can deduce the extinction $A_{[\mathrm{OII}]}$ at the [OII] wavelength. The values for $A_{[\mathrm{OII}]}$ are indicated in Table 1, along with the visible extinction $A_{V}$ and the extinction $A_{\mathrm{H} \alpha}$ at $\mathrm{H} \alpha$ wavelength, obtained from the SED curves. The $A_{\mathrm{H} \alpha} / A_{[\mathrm{OII}]}$ ratio varies up to $10 \%$ from galaxy to galaxy but remains comparable to the value predicted by O'Donnell (1994) for a diffuse interstellar medium: $A_{\mathrm{H} \alpha} / A_{[\mathrm{OII}]}=1.86$. Since this ratio should remain constant for the different galaxies, the variations give an idea of the quality of the kcorrect fit. The obtained extinctions are in approximate agreement with observations (e.g. Förster Schreiber et al. 2011), and the SFRs deduced from the [OII] luminosity are given in Table 1 as SFR $_{[\mathrm{OII}]}$.

Moustakas et al. (2006) present an alternative empirical [OII] SFR calibration parametrized in terms of the $B$-band luminosity. This calibration is intended to remove, on average, the systematic effects of reddening and metallicity, as well as to reduce the scatter in the resulting SFR values. The SFR of the four galaxies studied here were also determined from UV and IR luminosities by Tacconi et al. (2013). Because UV light directly traces unobscured star formation and IR $24 \mu \mathrm{m}$ emission originates in small dust grains mainly heated by UV photons from young stars, the combination of the two is a sensitive tracer of the global SFR (Leroy et al. 2008, 2012). Tacconi et al. (2013) derive the global SFR of the four galaxies studied here from a combination of $\mathrm{UV}$ and Spitzer $24 \mu \mathrm{m}$ luminosities with the methods of Wuyts et al. (2011b). The SFR obtained through these two calibrations are given for information in Table 1 . The values differ up to $3 \sigma$ between the three methods, which gives an idea of the uncertainty of the SFR measurements. Since it gives a lower standard deviation, we use the Kewley et al. (2004) calibration in 

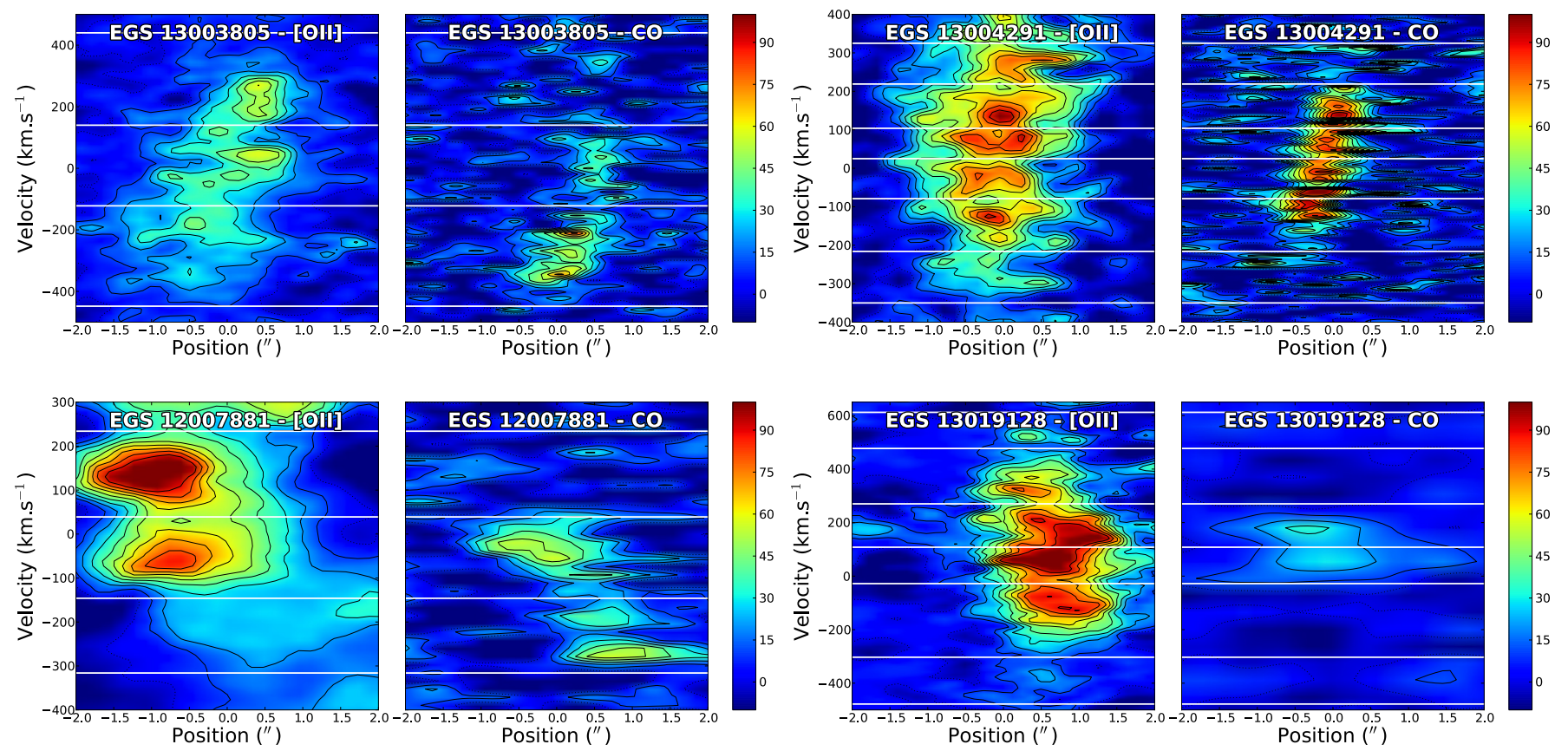

Fig. 2. [OII] line and CO luminosities (respectively left and right panels for each galaxy) in position-velocity planes corresponding to the DEEP2 slits. Smoothed ensembles of clumps are separated by eye along the vertical axis, as shown with the white horizontal lines. The [OII] diagrams were normalized in order to have fluxes proportional to the SFR, but all galaxies share the same arbitrary color scales. One arcsecond corresponds approximately to $8.5 \mathrm{kpc}$ (Table 1).

our study. However, [OII] measurements may not reveal some dust-embedded star forming regions, which probably explains the higher values of the SFR traced by UV and IR luminosities determined by Tacconi et al. (2013).

\section{A resolved Kennicutt-Schmidt law}

HST images of Fig. 1 reveal kpc-sized clumps within diffuse regions, but these clumpy features $\left(\sim 0.1^{\prime \prime}\right)$ are smoothed out at DEEP2 and IRAM resolutions, whose ranges are respectively $0.6-1.0^{\prime \prime}$ and $0.5-1.6^{\prime \prime}$. Spectroscopy, however, helps separate different components, thanks to their kinematics. DEEP2 spectra correspond to position-velocity diagrams (PV diagrams) along the galaxy major axis (Davis et al. 2007). Figure 2 compares the [OII] position-velocity diagrams with the corresponding slices in $\mathrm{CO}(3-2)$, both with the same $1^{\prime \prime}$ width. Smoothed $1^{\prime \prime}$-sized ensembles of clumps are separated by eye along the velocity axis of the PV diagrams. The characteristics of these ensembles are given in Table 2, and we aim at describing the star formation efficiency within them. We tried to compensate for the substructure separation by eye by taking all identifiable ensembles of clumps into account.

From the $\mathrm{CO}$ and $[\mathrm{OII}]$ lines, we estimate the gas mass and the SFR contained in areas of $1^{\prime \prime}$ in diameter (corresponding to the width of the slice and approximately to the size of the ensembles of clumps), and obtain the corresponding averaged surface densities, $\Sigma_{\text {gas }}$ and $\Sigma_{\text {SFR. }}$. As shown in Fig. 3, the depletion time $\left(t_{\text {depl }}=M_{\text {gas }} / \mathrm{SFR}\right)$ is equal to $1.9 \pm 0.3 \mathrm{Gyr}$, to be compared with results for samples of whole galaxies $(2.1 \mathrm{Gyr}$ at $z=0$ in Kennicutt 1998b, 0.5-1.5 Gyr from $z \sim 2$ to $z \sim 0$ in Genzel et al. 2010, 1.05 $\pm 0.74 \mathrm{Gyr}$ in Saintonge et al. 2011, $\sim 0.7 \mathrm{Gyr}$ at $z=1-3$ in Tacconi et al. 2013) and samples of subgalactic regions at low redshift $(2.0 \pm 0.8$ Gyr in Bigiel et al. 2008 and 2.35 Gyr in Bigiel et al. 2011). The resulting KS diagram is displayed in Fig. 4. The data points scatter around the line of constant depletion time equal to $1.9 \mathrm{Gyr}$ (such a line corresponding to a power law $\Sigma_{\mathrm{SFR}} \propto \Sigma_{\text {gas }}^{N}$ of exponent $N=1$ ). However, the depletion time is locally very different from one data point to the other, suggesting that the star formation scaling laws are different from one ensemble of clumps to the next within a galaxy. The scatter is comparable to the $\sim 0.3$ dex scatter observed for resolved local galaxies (Bigiel et al. 2008, 2011). The corresponding values obtained with the SFR from Tacconi et al. (2013) and through the $B$-band calibration of Moustakas et al. (2006) are indicated in Table 3, and give an idea of the uncertainties due to the SFR calibration method. Our sample is too incomplete to compute a best fit slope, but the method developed here could be applied to more high redshift galaxies.

\section{Discussion and conclusion}

\subsection{Advantages of the method}

We have shown how the various ensembles of clumps can be separated by their kinematics in PV diagrams, even though the angular resolution of molecular gas and SFR data was not able to separate them in integrated intensity. As such, the KS diagram can be obtained within regions of the resolution size $\left(\sim 1^{\prime \prime}\right)$.

Previous resolved KS work at high redshift was only obtained using serendipitous amplification by gravitational lenses. Decarli et al. (2012) carried out one of the first spatially resolved studies in high redshift galaxies, using [NII], FIR, and $\mathrm{CO}$ observations for two gravitationally lensed $z \sim 3.9$ galaxies. They obtain a steep relation of slope $N=1.4 \pm 0.2$ between the dust continuum and the molecular gas surface brightness. Strong lenses are rare, and determination of the clumps physical parameters depend on the lensing model. Our method is probably more appropriate for a systematic study of the star formation at high redshift, until higher resolution instruments resolve the clumps.

In the absence of high resolution molecular gas data, Swinbank et al. (2012) report adaptive optics H $\alpha$ observations of eleven kpc-scale star forming regions identified in $z=0.84-2.23$ 
Table 2. Some properties of the clumps of EGS 13004291, EGS 13003805, EGS 12007881, and EGS 13019128 obtained from measurements in the position-velocity diagrams.

\begin{tabular}{lcccccc}
\hline \hline Clump & $M_{\text {gas }}\left[10^{10} M_{\odot}\right]^{1}$ & SFR $\left[M_{\odot} \mathrm{yr}^{-1}\right]^{2}$ & $\log _{10}\left(\frac{\Sigma_{\text {gas }}}{M_{\odot} \mathrm{pc}^{-2}}\right)^{3}$ & $\log _{10}\left(\frac{\Sigma_{\mathrm{SFR}}}{M_{\odot} \mathrm{yr}^{-1} \mathrm{kpc}^{-2}}\right)^{3}$ & $t_{\mathrm{depl}}[\mathrm{Gyr}]^{4}$ & $F W H M\left[^{\prime \prime}\right]^{5}$ \\
\hline EGS 13003805-I & 11.85 & 38.23 & 3.019 & -0.473 & 3.10 & 0.93 \\
EGS 13003805-II & 6.92 & 34.75 & 2.785 & -0.514 & 1.99 & 0.78 \\
EGS 13003805-III & 3.18 & 24.99 & 2.447 & -0.657 & 1.27 & 0.63 \\
\hline EGS 13004291-I & 2.37 & 11.37 & 2.324 & -0.995 & 2.09 & 0.78 \\
EGS 13004291-II & 5.80 & 27.08 & 2.713 & -0.618 & 2.14 & 0.64 \\
EGS 13004291-III & 7.75 & 26.16 & 2.838 & -0.633 & 2.96 & 0.74 \\
EGS 13004291-IV & 4.96 & 23.92 & 2.644 & -0.672 & 2.07 & 0.79 \\
EGS 13004291-V & 6.28 & 33.83 & 2.761 & -0.522 & 1.92 & 0.61 \\
EGS 13004291-VI & 3.99 & 23.45 & 2.550 & -0.681 & 1.70 & 1.1 \\
\hline EGS 12007881-I & 5.33 & 25.38 & 2.680 & -0.642 & 2.10 & 1.35 \\
EGS 12007881-II & 7.26 & 41.88 & 2.814 & -0.425 & 1.73 & 1.36 \\
EGS 12007881-III & 1.41 & 51.05 & 2.10 & -0.339 & 0.28 & 1.57 \\
\hline EGS 13019128-I & 1.70 & 4.03 & 2.165 & -1.460 & 4.22 & 0.83 \\
EGS 13019128-II & 0.58 & 57.23 & 1.697 & -0.307 & 0.10 & 1.14 \\
EGS 13019128-III & 7.08 & 52.78 & 2.785 & -0.342 & 1.34 & 1.67 \\
EGS 13019128-IV & 5.25 & 47.51 & 2.655 & -0.388 & 1.10 & 1.60 \\
EGS 13019128-V & 0.78 & 32.32 & 1.828 & -0.555 & 0.24 & 1.62 \\
EGS 13019128-VI & 2.60 & 5.89 & 2.351 & -1.295 & 4.42 & 1.86 \\
\hline
\end{tabular}

Notes. ${ }^{(1)}$ The gas mass obtained as a fraction of the total mass of the galaxy from the CO position-velocity diagram; ${ }^{(2)}$ the SFR obtained similarly from the [OII] position-velocity diagram, normalized with the total SFR determined according to Sect. 2.2; ${ }^{(3)}$ the derived gas mass and SFR surface densities averaged over the same area of $1^{\prime \prime}$ in diameter; ${ }^{(4)}$ the depletion time $t_{\text {depl }}=M_{\text {gas }} /$ SFR; and ${ }^{(5)}$ the FWHM obtained from the IRAM position-velocity diagram with a Gaussian fit in the direction of the slice (for information only). The FWHM roughly corresponds to the IRAM beam size, so we preferred to use the same 1" size for all clumps in the different calculations. Using the FWHM instead of a constant $1^{\prime \prime}$ diameter to calculate the surface densities gives a much larger scatter, but actually does not change the constant depletion time fit and the values of $t_{\text {depl }}$. The clumps are numbered from bottom to top according to the horizontal separation lines of Fig. 2.

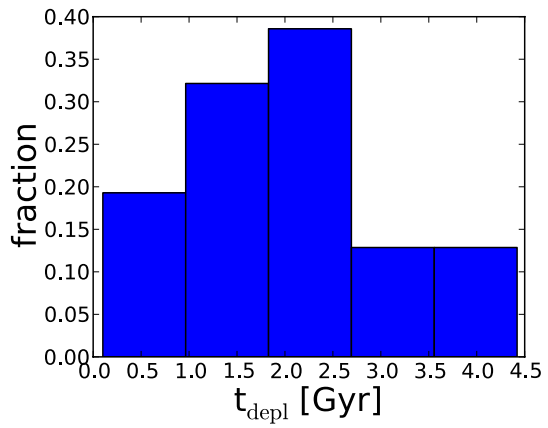

Fig. 3. Distribution of the depletion time for the 16 regions identified in the four galaxies studied here, using the Kewley et al. (2004) [OII] SFR calibration. The distribution has a mean of $t_{\mathrm{depl}}=1.9 \mathrm{Gyr}$ and a standard deviation of $1.2 \mathrm{Gyr}$, for a median value of $2.0 \mathrm{Gyr}$. Since we identified 18 ensembles of clumps, the standard error of the mean is $0.3 \mathrm{Gyr}$.

galaxies and measure the velocity dispersion of the ionized gas $\sigma$ and the star formation surface density $\Sigma_{\mathrm{SFR}}$. By assuming that $\sigma$ also corresponds to the dispersion of the cold clumps and that the clumps are marginally stable with a Toomre parameter $Q \simeq 1$, they claim a correlation between the gas surface density $\Sigma_{\text {gas }}$ and $\Sigma_{\text {SFR }}$. But the method is highly indirect, relies on many assumptions, and underestimates beam-smearing effects in the determination of $\sigma$.

\subsection{Biases and uncertainties}

The four galaxies studied here are particularly luminous and were selected from a sample (Tacconi et al. 2010) with stellar masses and star formation rates that are respectively higher than $3 \times 10^{10} M_{\odot}$ and $40 M_{\odot} \mathrm{yr}^{-1}$. A high luminosity was also needed

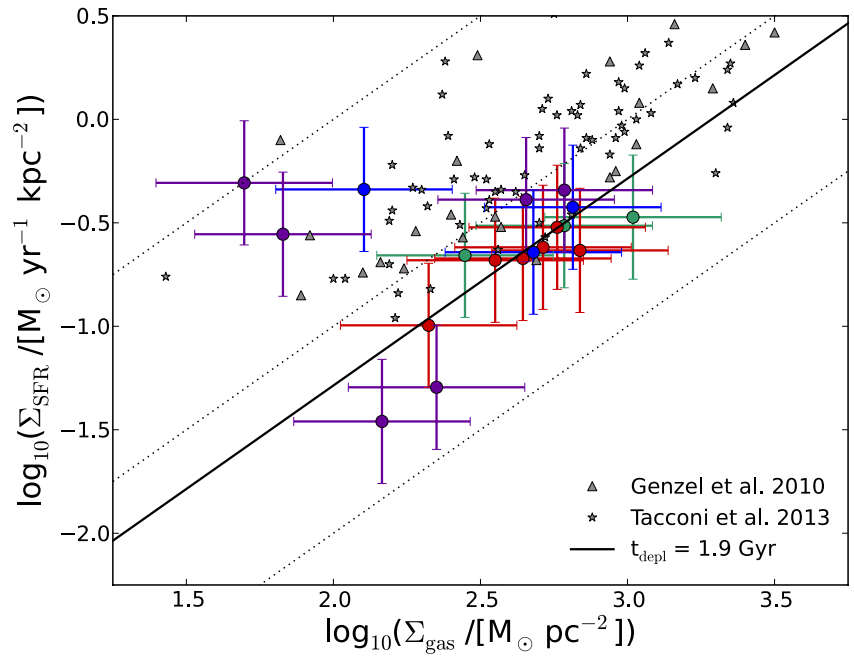

Fig. 4. Kennicutt-Schmidt diagram for 1 " ensembles of clumps of EGS13003805 (green), EGS13004291 (red), EGS12007881 (blue), and EGS13019128 (purple), using the Kewley et al. (2004) [OII] SFR calibration. The dotted diagonal lines correspond to constant gas depletion times of $0.1,1$, and $10 \mathrm{Gyr}$ from top to bottom, and the solid black line to a constant depletion time equal to the mean depletion time of the clumps, $t_{\mathrm{depl}}=1.9 \mathrm{Gyr}$. The error bars of 0.3 dex yield a reduced $\chi^{2}$ close to 1 and correspond to a factor 2 uncertainty, which is a lower estimate. The gray data points from Genzel et al. (2010) and Tacconi et al. (2013) are indicated for comparison with whole galaxies.

to visualize and isolate the clumps in the PV diagrams, so this method is intrinsically biased towards massive galaxies.

The main uncertainties for estimating gas and SFR surface densities come from the SFR calibration and from the values used for $\alpha=M_{\mathrm{H}_{2}} / L_{\mathrm{CO}}^{\prime}$, for the $\mathrm{CO}(3-2) / \mathrm{CO}(1-0)$ luminosity 
Table 3. Mean, median, and standard deviation of the depletion time of the clumps obtained through three different calibrations of the SFR.

\begin{tabular}{lccc}
\hline \hline \multirow{2}{*}{ SFR calibration } & \multicolumn{3}{c}{ Depletion time [Gyr] } \\
\cline { 2 - 4 } & mean & median & std. dev. \\
\hline Kewley et al. (2004) & 1.9 & 2.0 & 1.2 \\
Tacconi et al. (2013) & 2.1 & 0.7 & 2.9 \\
Moustakas et al. (2006) & 1.8 & 1.6 & 1.3 \\
\hline
\end{tabular}

Notes. Kewley et al. (2004) [OII] calibration, UV $+24 \mu \mathrm{m}$ calibration from Tacconi et al. (2013) and Moustakas et al. (2006) [OII] B-band calibration.

ratio, and for the extinction $A_{\mathrm{H} \alpha}$. These quantities could vary significantly from one galaxy to another and within each galaxy, thus increasing the scatter and the uncertainty of our measurements. For example, the $\mathrm{CO}(3-2)$ transition is less directly related to the molecular gas mass than the $\mathrm{CO}(1-0)$, and variations in the $\mathrm{CO}$ luminosity ratios as high as of a factor $\sim 2$ can be observed within a single galaxy (e.g. Koda et al. 2013). Significant variations in extinction values from one substructure to another within each galaxy are also expected, as observed in local galaxies (e.g. Scoville et al. 2001) and high redshift galaxies. Genzel et al. (2013) notably uses the $\mathrm{CO}(3-2)$ and $\mathrm{H} \alpha$ lines, as well as HST multiband images of a high redshift galaxy belonging to the same sample as ours to show that the methodology used to correct for extinction has a certain influence on the shape of the KS relation, especially on its slope. We thus expect that using a resolved extinction map instead of a single value would significantly change the depletion time we obtain. Nevertheless, identifying the ensembles of clumps in PV diagrams and the low spatial resolution of the [OII] and CO measurements prevent us from finding the corresponding structures in two-dimensional images and deriving extinction maps for our ensembles of clumps. As seen in Sect. 2.2, the values of the SFR are highly dependent on the calibration and, since the scatter in the observed $[\mathrm{OII}] / \mathrm{H} \alpha$ flux ratio is always higher than $32 \%$ (Moustakas et al. 2006), this remains a lower limit of the uncertainty in the SFR determined from [OII]. We expect our final values for the mass of gas and the SFR to be determined with uncertainty factors at least as high as 2 or 3 .

\subsection{Conclusion}

Our results, as well as most other observations (Genzel et al. 2010; Tacconi et al. 2010, 2013; Decarli et al. 2012; Swinbank et al. 2012), indicate that the star formation scaling law between SFR and gas surface densities is not significantly different at high redshift than in the local Universe. Our limited sample of $\sim 8 \mathrm{kpc}$-scale ensembles of clumps of distant galaxies is compatible with a constant depletion time of $1.9 \mathrm{Gyr}$, which is of the same order of magnitude as measurements at lower redshift. This adds to the growing evidence that the star formation processes ten billion years ago were similar to the ones that are observed in the local Universe. The method developed here could be applied to a more significant sample of high redshift galaxies to obtain more statistically robust results.

Acknowledgements. J. Freundlich acknowledges support by the École Normale supérieure (ENS, Paris), and is thankful to Philippe Salomé for numerous technical tips and Martin Stringer for his proofreading and suggestions. The authors wish to thank the anonymous referee, whose comments have led to significant improvements in this paper. This work is based on observations carried out with the IRAM Plateau de Bure Interferometer. IRAM is supported by INSU/CNRS (France), MPG (Germany) and IGN (Spain). This work also makes use of data from AEGIS, a multiwavelength sky survey conducted with the Chandra, GALEX, Hubble, Keck, CFHT, MMT, Subaru, Palomar, Spitzer, VLA, and other telescopes and supported in part by the NSF, NASA, and the STFC.

\section{References}

Bigiel, F., Leroy, A., Walter, F., et al. 2008, ApJ, 136, 2846

Bigiel, F., Leroy, A., Walter, F., et al. 2011, ApJ, 730, L13

Blanton, M. R., \& Roweis, S. 2007, ApJ, 133, 734

Bournaud, F., \& Elmegreen, B. C. 2009, ApJ, 694, L158

Coil, A. L., Newman, J. A., Kaiser, N., et al. 2004, ApJ, 617, 765

Cooper, M. C., Aird, J. A., \& Coil, A. L. 2011, ApJS, 193, 14

Cooper, M. C., Newman, J. A., Davis, M., et al. 2012, ASCL, 1203.003

Daddi, E., Dickinson, M., Morrison, G., et al. 2007, ApJ, 670, 156

Davis, M., Faber, S. M., Newman, J. A., et al. 2003, Proc. SPIE, 4834, 161

Davis, M., Guhathakurta, P., Konidaris, N. P., et al. 2007, ApJ, 660, L1

Decarli, R., Walter, F., Neri, R., et al. 2012, ApJ, 752, 2

Dekel, A., Birnboim, Y., Engel, G., et al. 2009a, Nature, 457, 451

Dekel, A., Sari, R., \& Ceverino, D. 2009b, ApJ, 703, 785

Dickman, R. L., Snell, R. L., \& Schoerb, F. P. 1986, ApJ, 309, 326

Faber, S. M., Phillips, A. C., Kibrick, R. I., et al. 2002, Proc. SPIE, 4841, 1657

Förster Schreiber, N. M., Shapley, A. E., Genzel, R., et al. 2011, ApJ, 739, 45

Genzel, R., Tacconi, L. J., Gracia-Carpio, J., et al. 2010, MNRAS, 407, 2091

Genzel, R., Tacconi, L. J., Kurk, J., et al. 2013, ApJ, submitted [arXiv: 1304.0668]

Kennicutt, R. C. Jr. 1998a, ApJ, 498, 541

Kennicutt, R. C. Jr. 1998b, ARA\&A, 36, 189

Kereš, D., Katz, N., Weinberg, D. H., \& Davé, R. 2005, MNRAS, 363, 2

Kewley, L. J., Geller, M. J., \& Jansen, R. A. 2004, ApJ, 127, 2002

Koda, J., Scoville, N., Hasegawa, T., et al. 2012, ApJ, 761, 41

Leroy, A. K., Walter, F., Brinks, E., et al. 2008, ApJ, 136, 2782

Leroy, A. K., Bigiel, F., de Blok, W. J. G., et al. 2012, ApJ, 144, 3

Leroy, A. K., Walter, F., Sandstrom, K., et al. 2013 [arXiv: 1301.2328]

Mannucci, F., Cresci, G., Maiolino, R., et al. 2010, MNRAS, 408, 2115

Moustakas, J., Kennicutt, R. C., \& Tremonti, C., 2006, ApJ, 642, 775

Newman, J. A., Cooper, M. C., Davis, M., et al. 2012, ApJS, submitted [arXiv: 1203.3192]

Noeske, K. G., Weiner, B. J., Faber, S., et al. 2007, ApJ, 660, L43

O’Donnell, J. E. 1994, ApJ, 422, 158

Renaud, F., Kralkjic, K., \& Bournaud, F. 2012, ApJ, 760, L16

Saintonge, A., Kauffmann, G., Wang, J., et al. 2011, MNRAS, 415, 61

Schlegel, D., Finkbeiner, D., \& Davis, M. 1998, ApJ, 121, 161

Scoville, N. Z., Polletta, M., Ewald, S., et al. 2001, ApJ, 122, 3017

Solomon, P. M., Rivolo, A. R., Barrett, J., et al. 1987, ApJ, 319, 730

Solomon, P. M., Downes, D., Radford, S. J. E., et al. 1997, ApJ, 478, 144

Swinbank, A. M., Smail, I., Sobral, D., et al. 2012, ApJ, 760, 130

Tacconi, L. J., Genzel, R., Neri, R., et al. 2010, Nature, 463, 781

Tacconi, L. J., Neri, R., Genzel, R., et al. 2013, ApJ, 768, 74

Van de Voort, F., Schaye, J., Booth, C. M., et al. 2011, MNRAS, 414, 2458

Wyder, T. K., Martin, D. C., Barlow, T. A., et al. 2009, ApJ, 696, 1834

Wuyts, S., Förster Schreiber, N., Lutz, D., et al. 2011a, ApJ, 738, 106

Wuyts, S., Förster Schreiber, N. M., van der Wel, A., et al. 2011b, ApJ, 742, 96 